\title{
Color Reduction using the Combination of the Kohonen Self-Organized Feature Map and the Gustafson-Kessel Fuzzy Algorithm
}

\author{
Konstantinos Zagoris, Nikos Papamarkos* and Ioannis Koustoudis \\ *Image Processing and Multimedia Laboratory \\ Department of Electrical \& Computer Engineering \\ Democritus University of Thrace \\ 67100 Xanthi, Greece, papamark@ee.duth.gr
}

\begin{abstract}
The color reduction in digital images is an active research area in digital image processing. In many applications such as image segmentation, analysis, compression and transmission, it is preferable to have images with a limited number of colors. In this paper, a color clustering technique which is a combination of a Kohonen Self Organized Featured Map (KSOFM) and a fuzzy clustering algorithm is proposed. Initially, we reduce the number of image's colors by using a KSOFM. Then, using the KSOFM color clustering results as starting values, we obtain the final colors by a Gustafson-Kessel Fuzzy Classifier (GKFC). Doing this, we lead to better color classification results because the final color classes obtained are not spherical.

Keywords: Color Reduction, Color Clustering, Neural Networks, Fuzzy Clustering.
\end{abstract}

\section{Introduction}

Nowadays the color of the digital images is one of the most widely used information for the image processing researchers. Digital images are usually described by a set of pixels uniformly distributed in a two-dimensional grid. In color images each color is expressed by a vector containing the values of three color components. True-type color images consist of more than 16 million different colors, in a 24-bit RGB color space. However, in many applications, such as image segmentation, analysis, compression and transmission, it is preferable to have images with a limited number of colors. The objective of color reduction is to cluster the $N$ colors of an image into only c color 
clusters, where $c<N$. Several techniques have been proposed in the literature for color reduction. Firstly, there is a group of techniques that repeatedly divide the 3D color distribution in disjoint regions [1]. The methods of Octree [2,3], median-cut [4] and variance-based algorithm [5] are some of those splitting algorithms.

The second major class of algorithms is based on cluster analysis in the color space. Techniques in this category attempt to find the optimal palette using vector classifiers like the Growing Neural Gas (GNG) [6], Adaptive Color Reduction [7], FOSART [8$11]$, Fuzzy ART $[12,13]$ and FCM [14].

Techniques in the third category are general color segmentation techniques which can be considered as color reduction algorithms. For instance, the mean-shift based procedures for feature space analysis have been also used for color redaction [15-16].

Most of the above clustering techniques lead to spherical clusters. This is an important disadvantage in cases where we have dominant colors with non-spherical color distribution. One way to overcome this problem is to use a classifier, which using a non-Euclidean distance, leads to ellipsoidal classes. One such classifier is the GKFC which uses the Mahalanobis distance. However, this classifier has two main disadvantages, that is, the final results depends on the initial cluster, and also, it has high computation cost. To overcome these difficulties, in this paper we propose a new color clustering technique which is based on a combination of a KSOFM neural network and a GKFC. Initially, we perform color reduction by using the KSOFM. After this, we have a number of color classes and each initial color is classified to one of these classes. In the final stage, the KSOFM results are considered as starting values and fed the GKFC. The final clustering results obtained by the GTFC give us the color palette of the final image. It should be noticed that an initial version of this paper is published in MLDM 2007/ICDM proceedings [17].

The paper is organized as follows. Section 2 describes shortly the KSOFM neural network. Section 3 describes the GKFC as it is used in this work. Section 4 provides details of our method for the color reduction technique. Section 5 presents the experimental and comparative results and finally Section 6 conclude the paper.

\section{Kohonen Self Organized Featured Map}

A major category of neural networks is the self-organized neural networks which do not need supervising during their training phase. Their goal is to distinguish patterns in their training data and separate them in groups. The KSOFM [18,19] invented by the Prof. Teuvo Kohonen, is the most famous and widely used self-organized neural network. It is a single layer feedforward network but it differs in how it is trained and in how it revokes a pattern.

Analytically, the Kohonen network consists of two layers, the input and the competitive layer. In the latter layer the output units are arranged in one, two or higher dimensional grids. As depicted in the architecture of the KSOFM shown in Fig. 1, each input class has a feedforward connection to each one of the output classes. So, the neural network maps a set of input vectors into a set of output vectors without supervision. The knowledge the network acquires by the training data is stored in the weights $w_{j k}$. These 
weights express the extent to which the connection of the data $x_{k}$ with the output neuron $y_{j}$ is important.

In the proposed method the input features are the three color components of the RGB color space and the output units corresponds to the centers of the color classes which define the dominant color of each class. Therefore, the number of the output units is equal to the desirable amount of colors.

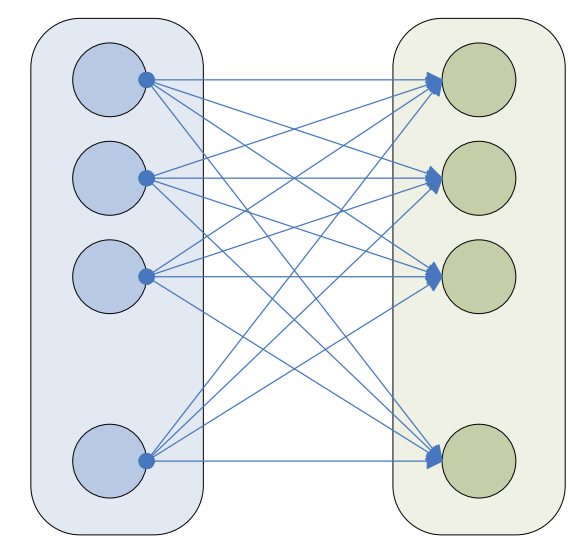

Fig. 1. The Architecture of the Kohonen Self-Organizing Map.

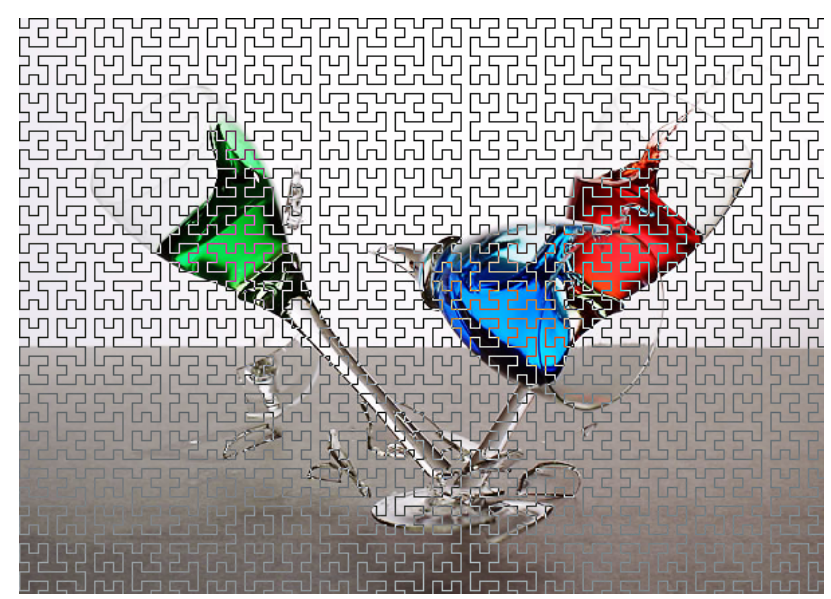

Fig. 2. The sub sampling of the image based on the Hilbert's space filling curve. 
The training algorithm of the KSOFM is based on competitive learning. Each time an input vector $x_{k}$ is presented, a winner output neuron $y_{j}$ is calculated based on the Euclidean Distance.

$$
y_{j}=\arg \min \left\|x_{k}-w_{j k}\right\|
$$

The winner output neuron changes its connections weights $w_{j k}$ as follows:

$$
\Delta w_{j k}=n\left(x_{k}-w_{j k}\right)
$$

The $n$ is a variable that defines the learning rate of the training process and it is not constant but changes each time a new input vector is presented. In this work, the learning rate takes the following values: $n_{\text {initially }}=10^{-2}, n_{\text {final }}=10^{-4}, n_{\text {step }}=10^{-5}$.

One of the most important parts of the training process is the selection of the samples data. It is preferable to have as training samples data a sub-sampling version of the original image instead of the whole image in order to achieve reduction of the computational time. In the proposed color reduction technique, the training samples are selected in a similar way as in ACR technique [7] from the peaks of the well-known Hilbert's space filling curve [20]. As the Fig. 2 depicts, the Hilbert's space filling curve is one dimensional curve which visits every possible pixel within a two dimensional space. This fractal scanning technique is much more efficient in capturing features in a digital image than the commonly used techniques of raster scanning because the neighborhood relation between the pixels is retained.

After the end of the training phase, the KSOFM initially is fed with unknown samples (all the pixels of the image) and then it correlates the samples to the centers of the classes that the neural net converged at the training phase.

\section{Gustafson - Kessel fuzzy algorithm}

\subsection{Overview}

One major problem of the standard fuzzy c-means algorithm is that it produces spherical classes. For example, if the sets of points presented at Fig. 3(a) pass through the fuzzy cmean algorithm for partitioning into four classes, the result will not be the optimal (Fig 3(b).

The Gustafson - Kessel [21] is an extension of the fuzzy c-means algorithm that deals with this problem by using the covariance matrix in order to detect ellipsoidal classes.

That is, each cluster is characterized by its center and its own-inducing matrix $A_{i}$ which forms the following distance for each class:

$$
d_{i k}^{2}=\left(x_{k}-v_{i}\right)^{T} A_{i}\left(x_{k}-v_{i}\right) .
$$

The $x_{k}, k \in[1, n]$ are the data vectors and the $v_{i}, i \in[1, c]$ are the centers of the classes. The $A_{i}$ is a positive-define matrix adapted to the topological structure of the 
data inside a cluster. The following equation (Eq. 4) indicates that the objective function of the Gustanfson - Kessel algorithm is linear in terms of $A_{i}$ and cannot be directly minimized with respect to it.

$$
J(U, V)=\sum_{i=1}^{c} \sum_{k=1}^{n} u_{i k}^{m} d_{i k}^{2}
$$

So the $A_{i}$ must be constrained. This is accomplished by constraining its determinant:

$$
\left|A_{i}\right|=\rho_{i}, \quad \rho_{i}>0 \forall i
$$

Without any prior knowledge, the $\rho_{i}$ is fixed at 1 for each cluster. Finally, the $A_{i}$ is calculated from the following equation:

$$
A_{i}=\sqrt[h]{\rho_{i} \operatorname{det}\left(F_{i}\right)} F_{i}^{-1}, \quad i \in[1, c]
$$

where $h$ represent the number of dimensions of the space that the data reside. Because the RGB color space has three dimensions, the value of $h$ is equal to 3 in this work. $F_{i}$ is the covariance matrix of class $i$ which shows how the samples are scattered inside a class:

$$
F_{i}=\frac{\sum_{k=1}^{n}\left(u_{i k}\right)^{m}\left(x_{k}-v_{i}\right)\left(x_{k}-v_{i}\right)^{T}}{\sum_{k=1}^{n}\left(u_{i k}\right)^{m}}, \quad i \in[1, c]
$$

The weighting parameter $m, m \in(1, \infty)$ influence the crispness or the fuzziness of the resulting partition between the classes. Worth noticing that if Eq. 6 and Eq. 7 substituted into Eq. 3, the outcome will be a squared Mahalanobis distance norm.

Finally, the $U=\left[u_{i k}\right]$ is called partition matrix and is defined as the grade of membership of $x_{k}$ to the cluster $i$ and it must satisfy the following constraints:

$$
\begin{gathered}
0 \leq u_{i k} \leq 1, \quad i \in[1, c] \text { and } k \in[1, n] \\
\sum_{i=1}^{c} u_{i k}=1, \quad k \in[1, n] \\
0<\sum_{k=1}^{n} u_{i k}<n, \quad i \in[1, c]
\end{gathered}
$$




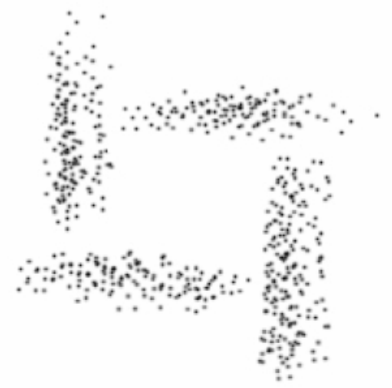

c.

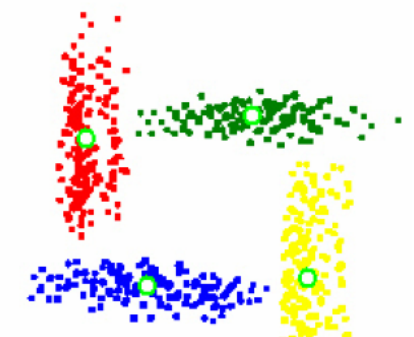

b.

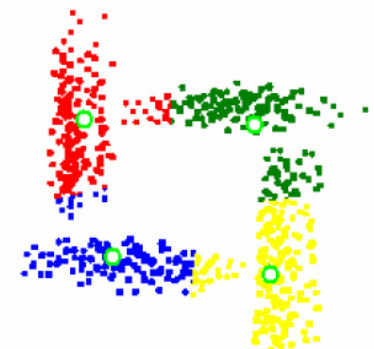

Fig. 3. (a) The points in the $2 \mathrm{D}$ space which must separate to four classes. (b) Four classes obtained by using the fuzzy c-means algorithm. (c) The four classes obtained through the Gustafson - Kessel algorithm.

\subsection{The main steps of the GKFC}

The Gustafson - Kessel algorithm consists of the following main steps:

Step 1: Define the number of the classes $c$, the weighting parameter $m$ and the cluster volumes $\rho_{i}$.

Step 2: Define the termination tolerance $\varepsilon>0$ and the number of iterations $\lambda$. Set a counter $\alpha$ equal to one ( $\alpha=1)$.

Step 3: Initialize randomly the partition matrix $U=\left[u_{i k}\right]$. In this work, the partition matrix is initialized not randomly but from the connections weights $w_{j k}$ of the KSOFM for each output class.

Step 4: Compute the centers of the classes $v_{i}$ according to the following equation: 


$$
v_{i}=\frac{\sum_{k=1}^{n}\left(u_{i k}\right)^{m} x_{k}}{\sum_{k=1}^{n}\left(u_{i k}\right)^{m}}, \quad i \in[1, c] \text { and } k \in[1, n]
$$

Step 5: Compute the covariance matrix $F_{i}$ for each class according to the Eq. 7 .

Step 6: Compute the matrix $A_{i}$ for each class according to Eq. 6.

Step 7: Compute the distance $d_{i k}$ of every sample $x_{k}$ from the center of each class $v_{i}$ according to the Eq. 3.

Step 8: Update the partition matrix $U=\left[u_{i k}\right]$ for each sample $x_{k}$ according to the following equation:

$$
u_{i k}=\frac{1}{\sum_{j=1}^{c}\left(\frac{d_{i k}}{d_{i j}}\right)^{\frac{2}{m-1}}}, \quad i \in[1, c] \text { and } k \in[1, n]
$$

However, in the extreme case where $d_{i k}=0$ for some $x_{k}$ and one or more classes $v_{i}$, the partition function $u_{i k}$ cannot be computed. In this case the 0 is assigned to each $u_{i k}$ while the rest of these that have $d_{i k}>0$ take arbitrary membership values but underlined to the constraint shown at the Eq. 9 .

Step 9: If $\max \left|U^{(\alpha)}-U^{(\alpha-1)}\right|<\varepsilon$ or $\alpha \geq \lambda$ then stop, else set $\alpha=\alpha+1$ and go to Step 4.

\section{Overview of the proposed method}

Figure 4 depicts the entire process of the proposed method. As it is mentioned above, it is a hybrid technique because it is based on the combination of the KSOFM and the GKFC. This technique is appropriate for reducing the colors of the image at a preprocessing stage for a segmentation technique. According to the description given in the previous section, the proposed color reduction technique consists of the following main steps:

Step 1: Assemble the training samples data from the sub-sampling of the original image from the peaks of the Hilbert's space filling curve.

Step 2: Define the desirable final number of colors for the image. This number defines also the output classes of the KSOFM and GKFC algorithm.

Step 3: Train the KSOFM by using the samples obtained from the sub-sampling procedure.

Step 4: Feed each color of the original image into the trained KSOFM. This maps (classify) each pixel to one of the output classes and ultimately to one of the reduced colors. 
Step 5: Initialize Gustafson - Kessel with the classification results of the KSOFM. That is, populate the partition matrix from the KSOFM connections weights of each output class.

Step 6: Run the Gustafson - Kessel algorithm and obtain the final values of the partition matrix.

Step 7: Using the clustering results defined by the GKFC reduce all the initial colors of the original image and obtain the final image with the predefined limited number of colors.

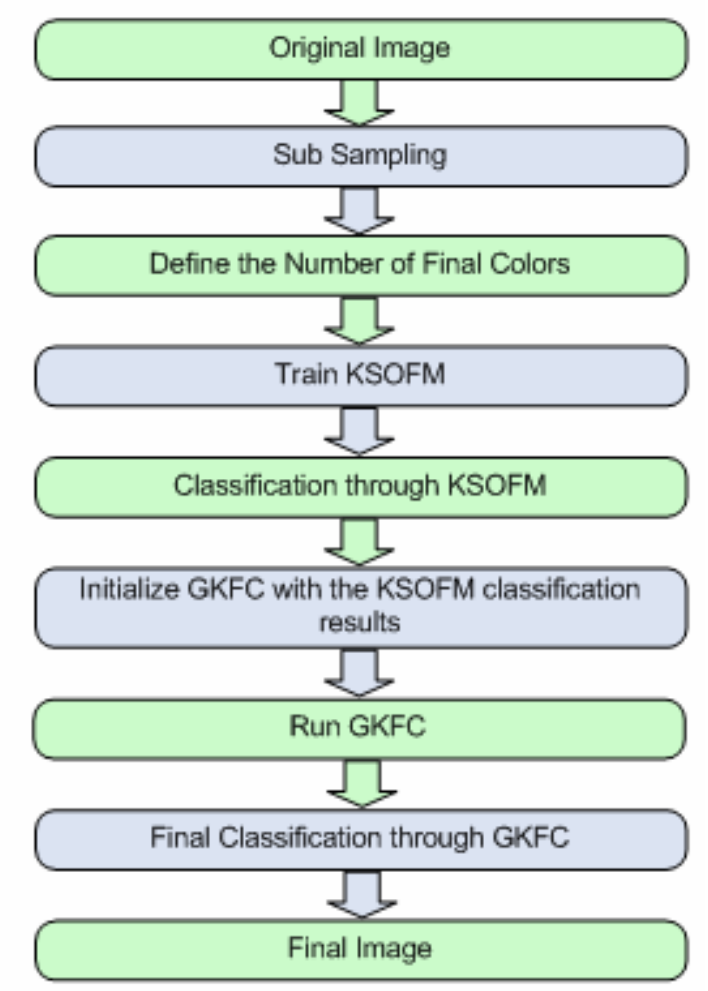

Fig. 4. The flow-chart of the proposed color reduction technique.

\section{Experimental Results}

The technique proposed in this paper is implemented with the help of a Visual Programming Environment (Borland Delphi). The program can be downloaded for testing at the web address: http://orpheus.ee.duth.gr/download/pythagoras.zip. 


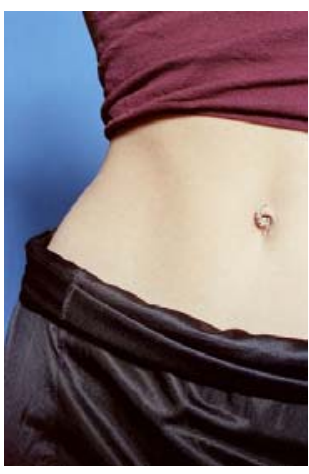

a.

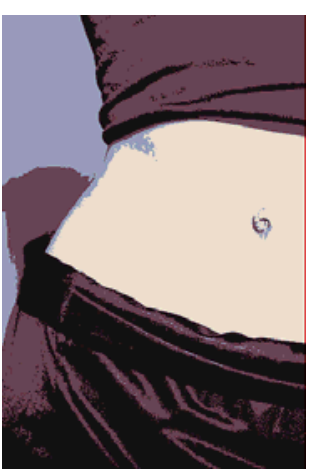

d.

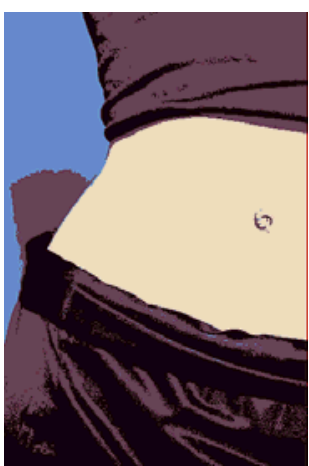

g.

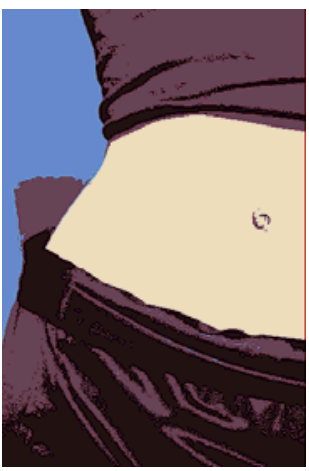

b.

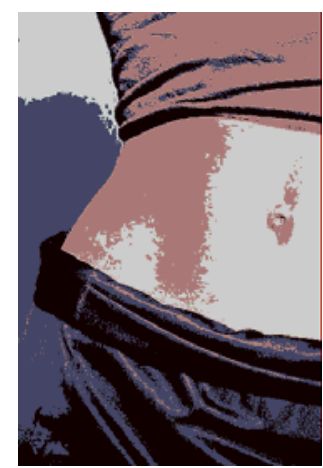

e.

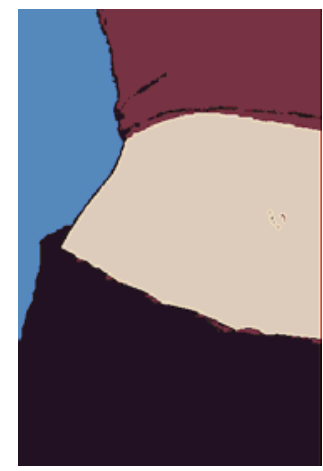

h.

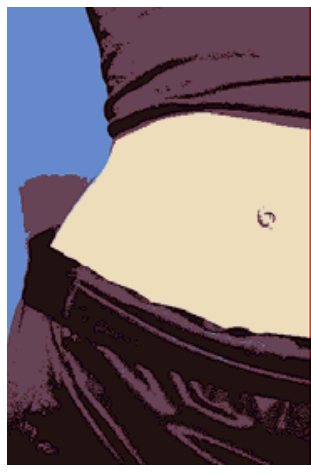

c.

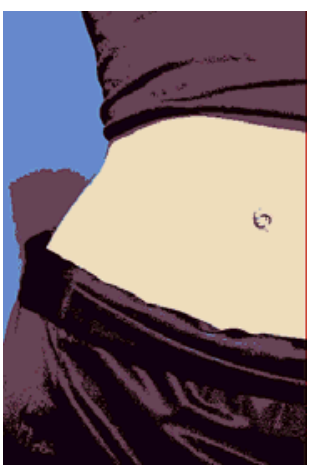

f.

Fig. 5. (a) The original image is constituted of 22410 colors. Final image obtained with only 4 colors by using: (b) KSOFM, (c) FCM, (d) GNG, (e) Median - Cut, (f) Wu Quantization, (g) Variance Quantization and (h) the proposed technique (KSOFM-GK). 
a.

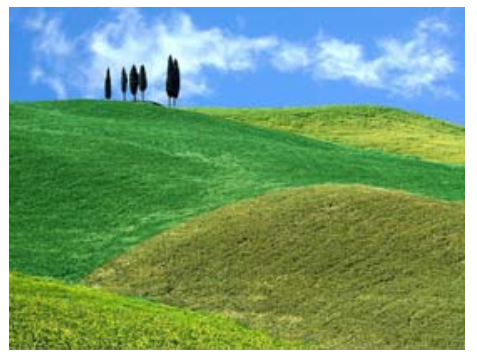

c.

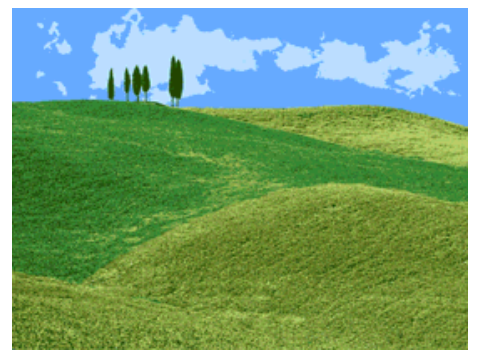

e.

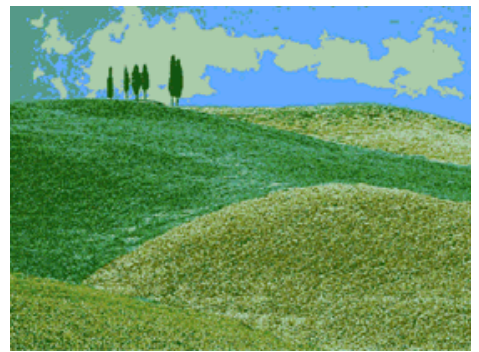

g.

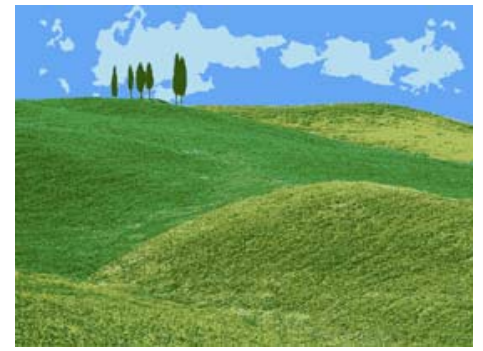

b.

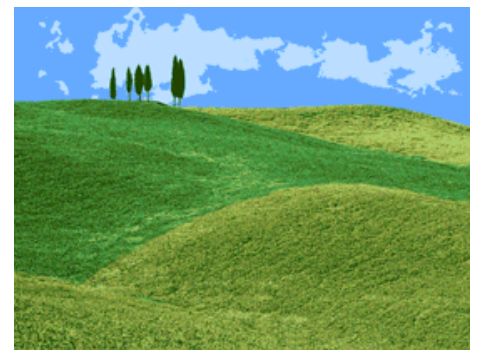

d.

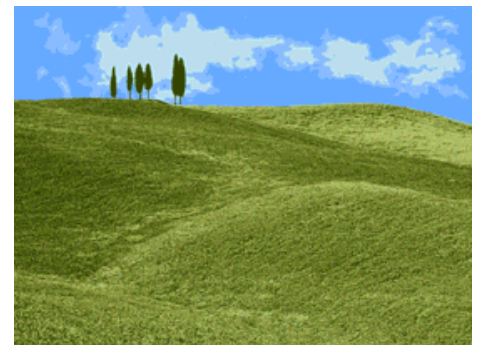

f.

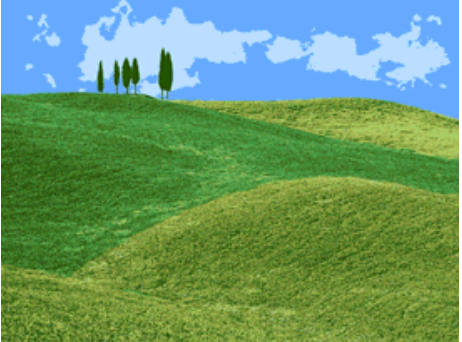

h.

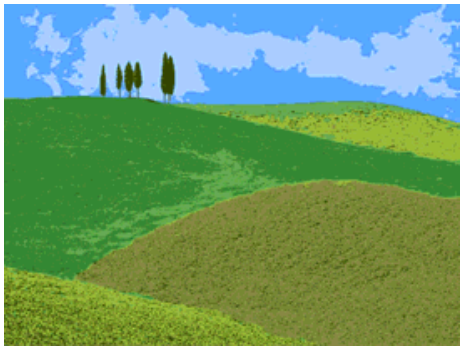

Fig. 6. (a) The original image has 99760 colors. Final image obtained with only 7 colors by using: (b) KSOFM, (c) FCM, (d) GNG, (e) Median - Cut, (f) Wu Quantization, (g) Variance Quantization and (h) the proposed technique (KSOFM-GK).

The proposed technique is tested on several images with satisfactory results. As the Figures 5, 6, 7, 8 and 9 depicts, the proposed method is compared with other popular color reduction techniques such as: KSOFM, FCM, GNG, Median - Cut, Wu Quantization and Variance Quantization. Table 1 presents the parameters of the algorithms during the testing. 
a.
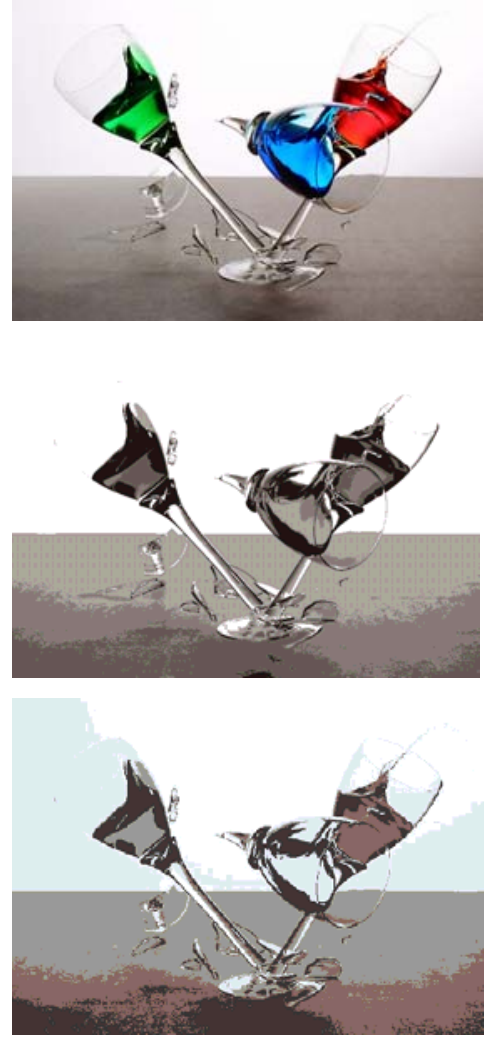

g.

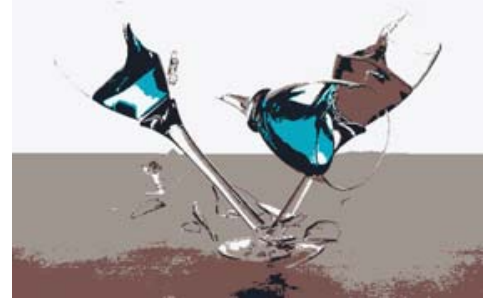

b.

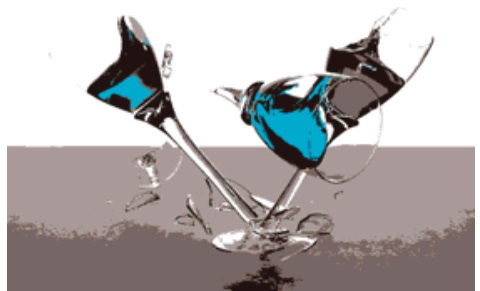

d.

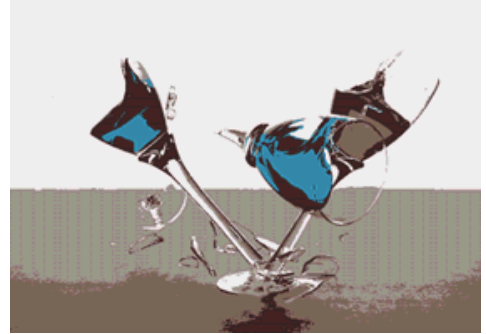

f.

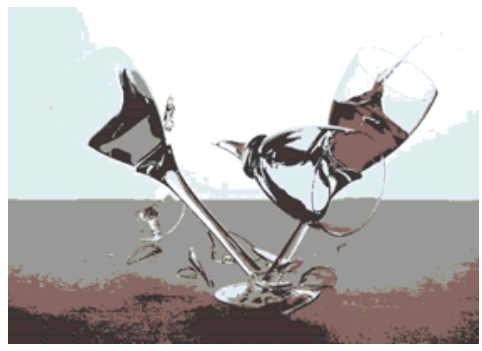

h.

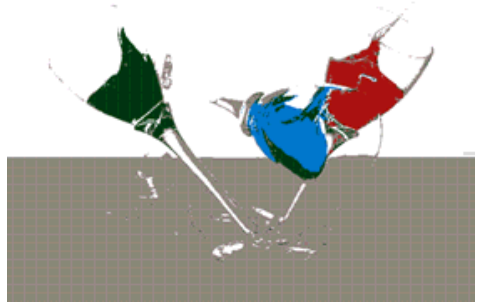

Fig. 7. (a) The original image is constituted of 33784 colors. Final image obtained with only 5 colors by using: (b) KSOFM, (c) FCM, (d) GNG, (e) Median - Cut, (f) Wu Quantization, (g) Variance Quantization and (h) the proposed technique (KSOFM-GK).

It is obvious that we cannot use for comparison SNR, PSNR or other similar criteria. The use of the GKFC leads to uniform color areas having well separated boundaries with the neighbor classes. For these reasons we have choose to apply the GKFC in the final stage of the proposed color reduction technique. Thus, the comparison of the proposed technique with other color reduction approaches cannot be based on statistical criteria but only on optical perception. 
a.

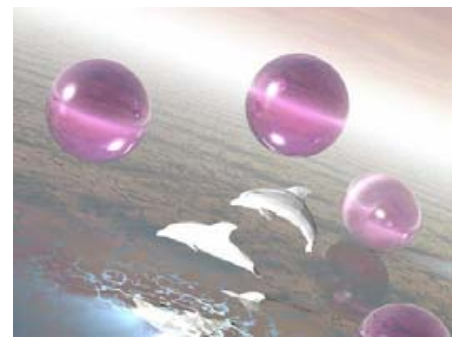

c.

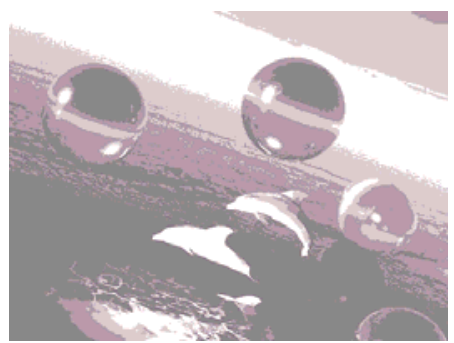

e.

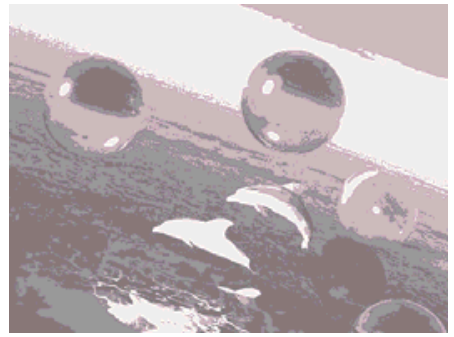

g.

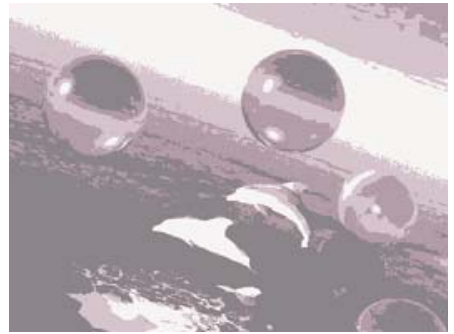

b.

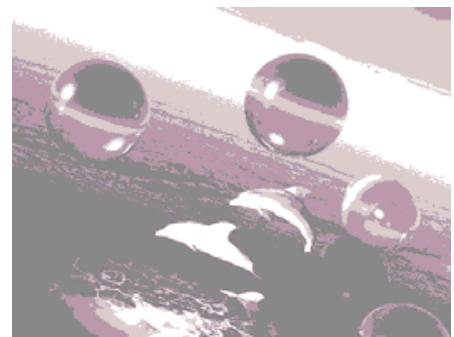

d.

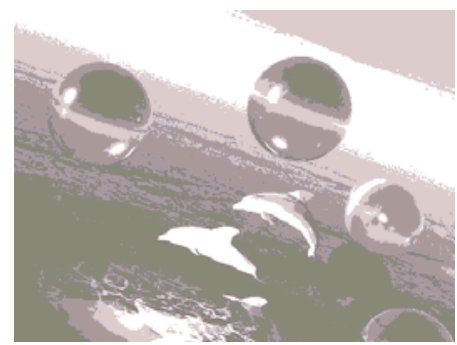

f.

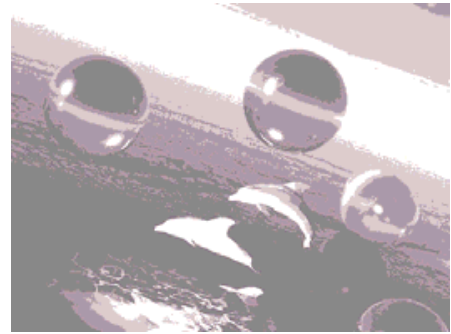

h.

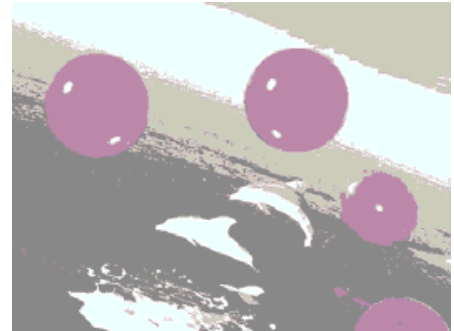

Fig. 8. (a) The original image has 31655 colors. Final image obtained with only 4 colors by using: (b) KSOFM, (c) FCM, (d) GNG, (e) Median - Cut, (f) Wu Quantization, (g) Variance Quantization and $(\mathrm{h})$ the proposed technique (KSOFM-GK).

The experimental results depicted in Figure 5 prove that the proposed technique manage to merge the colors of the clothes, skin and background better than the other techniques. The second experiment presented in Figure 6 demonstrates that the KSOFMGK technique preserved the dominant color of each different field and merged better their small color variations. The experiments of the Figures 7, 8 and 9 established the ability of the KSOFM-GK to maintain the dominants colors of the image: the colors of the glasses (Figure 7(h)), the color of the spheres (Figure 8(h)) and the color of the butterfly's feathers (Figure 9(h)). 
a.

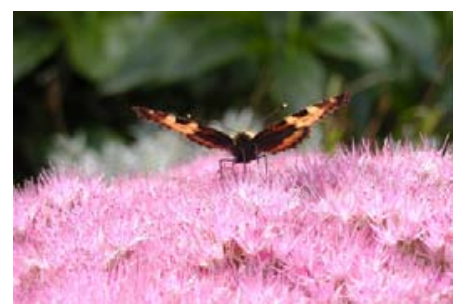

c.

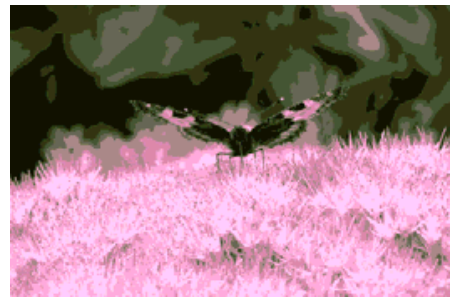

e.

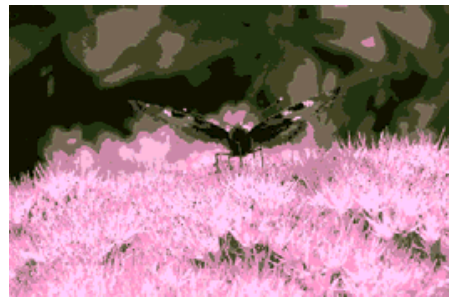

g.

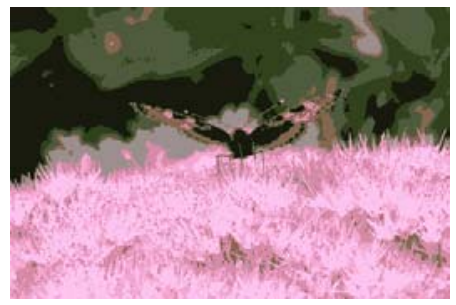

b.

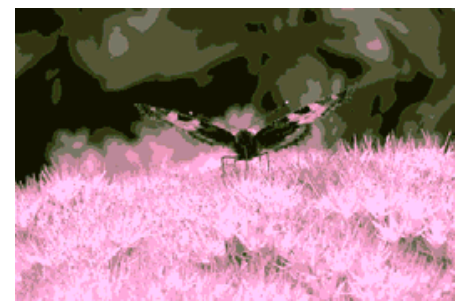

d.

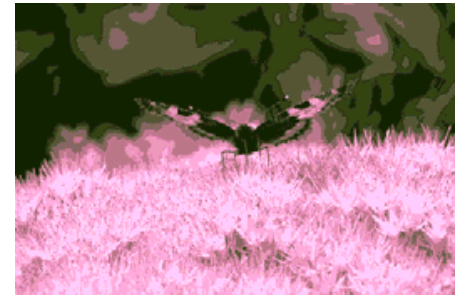

f.

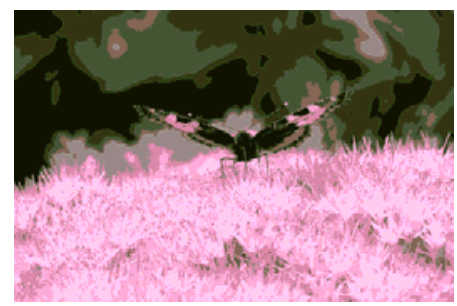

h.

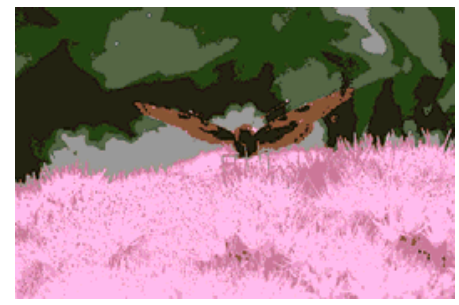

Fig. 9. (a) The original image has 69656 colors. Final image obtained with only 8 colors by using: (b) KSOFM, (c) FCM, (d) GNG, (e) Median - Cut, (f) Wu Quantization, (g) Variance Quantization and (h) the proposed technique (KSOFM-GK).

Generally, the experimental results have shown that the proposed technique has the ability to retain the dominant colors even if the final image consists of a very small number of unique colors. Also, it can merge areas of the image having similar colors. In this point of view, it can be considered as a powerful color image segmentation procedure.

\section{Conclusion}

In this paper an hybrid color reduction technique is proposed which is based on a combination of a KSOFM neural network and the Gustafson-Kessel fuzzy algorithm. 
Initially, the KSOFM is applied to the original image and produce a predefined number of color classes. Then, the final color reduction is performed by the Gustafson-Kessel fuzzy clustering algorithm considering the KSOFM clustering results as initial values. Thus, the entire clustering procedure can be considered as a hybrid neuro-fuzzy technique. The proposed method was tested in a variety of images and the results are compared to other color reduction techniques. The experimental results have shown the ability to retain the image's dominant colors. Also, it can merge areas of the image with similar colors producing uniform color areas. In this point of view the proposed technique can be used for color segmentation. Due to determination of the Mahalanobis distance in the GKFC the proposed technique has higher computational cost than others color reduction approaches. In order to speed up the entire technique, a fractal subsampling procedure based on the Hilbert's space filling curve is applied. The entire system is implemented in visual environment and it is available from http://orpheus.ee.duth.gr/download/pythagoras.zip.

Table 1. The parameters of the algorithms during the testing.

\begin{tabular}{|c|c|c|c|}
\hline KSOFM & Fuzzy C-Mean & $\overline{\mathrm{GNG}}$ & KSOFM -s GK \\
\hline $\begin{array}{l}\text { Initially Learning Rate: } \\
n_{\text {initially }}=10^{-2}\end{array}$ & $m=1.2$ & $\mathrm{ALPHA}=0.5$ & $\begin{array}{l}\text { Initially Learning } \\
\text { Rate: } n_{\text {initially }}=10^{-2}\end{array}$ \\
\hline $\begin{array}{l}\text { Final Learning Rate: } \\
n_{\text {final }}=10^{-4}\end{array}$ & Epochs $=2000$ & $B E T A=5 \cdot 10^{-4}$ & $\begin{array}{l}\text { Final Learning Rate: } \\
n_{\text {final }}=10^{-4}\end{array}$ \\
\hline Step of the Learning & Termination & Maximum Connection & Step of the Learning \\
\hline \multirow[t]{7}{*}{ Rate: $n_{\text {step }}=10^{-5}$} & $\begin{array}{l}\text { Tolerance: } \\
\varepsilon=5 \cdot 10^{-5}\end{array}$ & Age $=300$ & Rate: $n_{\text {step }}=10^{-5}$ \\
\hline & & $\begin{array}{l}\text { Learning Rate for the } \\
\text { Winner Neuron: }\end{array}$ & $\begin{array}{l}\text { KSOFM Termination } \\
\text { Tolerance: }\end{array}$ \\
\hline & & $n_{W}=2 \cdot 10^{-2}$ & $\varepsilon=5 \cdot 10^{-5}$ \\
\hline & & $\begin{array}{l}\text { Learning Rate for the } \\
\text { Neighboring Neuron: }\end{array}$ & $\begin{array}{l}\text { GKFC termination } \\
\text { Tolerance: }\end{array}$ \\
\hline & & $n_{N}=5 \cdot 10^{-4}$ & $\varepsilon=5 \cdot 10^{-4}$ \\
\hline & & Epochs $=2000$ & $m=1.2$ \\
\hline & & & Iterations: $\lambda=100$ \\
\hline
\end{tabular}

\section{Acknowledgments}

This paper is part of the 2003-03E $\Delta 679$ research project, implemented within the framework of the Reinforcement Programme of Human Research Manpower (PENED) and co-financed by National and Community Funds (25\%) from the Greek Ministry of Development - General Secretariat of Research and Technology and (75\%) from E.U. - European Social Fund. 


\section{References}

1. Scheunders, P.: A comparison of clustering algorithms applied to color image quantization. Pattern Recognit. Lett., vol. 18. (1997) 1379-1384

2. Ashdown, I.: Octree color quantization in Radiosity. A Programmer's Perspective. Wiley New York (1994)

3. Gervautz, M., Purgathofer, W.: A simple method for color quantization: Octree quantization. Graphics Gems, A. S. Glassner, Ed. Academic New York (1990) 287-293

4. Heckbert, P.: Color image quantization for frame buffer display. Comput. Graph., vol. 16 (1982) 297-307

5. Wan, S. J., Prusinkiewicz, P., Wong, S. K. M.: Variance based color image quantization for frame buffer display. Color Res. Applicat., vol. 15, no. 1 (1990) 52-58

6. Fritzke, B.: A growing neural gas network learns topologies. Tesauro, G., Touretzky, D.S., Leen, T.K. (Eds.), Advances in Neural Information Processing Systems, vol. 7. MIT Press, Cambridge, MA (1995) 625-632

7. Papamarkos, N., Atsalakis, A., Strouthopoulos, C.: Adaptive color reduction. IEEE Transactions on Systems, Man and Cybernetics Part B: Cybernetics 32 (2002)

8. Baraldi, A., Blonda, P.: A survey of fuzzy clustering algorithms for pattern recognition — part I. IEEE Transactions on Systems, Man, and Cybernetics-Part B: Cybernetics 29 (6) (1999) 778785

9. Baraldi, A., Blonda, P.: A survey of fuzzy clustering algorithms for pattern recognition-part II. IEEE Transactions on Systems, Man, and Cybernetics - Part B: Cybernetics 29 (6) (1999) 786801

10. Baraldi, A., Parmiggiani, F.: Novel neural network model combining radial basis function, competitive Hebbian learning rule, and fuzzy simplified adaptive resonance theory. Proceedings of the SPIE's Optical Science, Engineering and Instrumentation 1997: Applications of Fuzzy Logic Technology IV, vol 3165. San Diego CA (1997) 98-112

11. Baraldi, A., Parmiggiani, F.: A fuzzy neural network model capable of generating/ removing neurons and synaptic links dynamically. Blonda, P. Castellano, M. Petrosino, A. (Eds.) Proceedings of the WILF 1997-II Italian Workshop on Fuzzy Logic. World Scientific Singapore (1998) 247-259

12. Carpenter, G., Grossberg, S., Rosen, D. B.: Fuzzy ART: fast stable learning and categorization of analog patterns by an adaptive resonance system. Neural Networks 4. (1991) 759-771

13. Carpenter, G., Grossberg, S., Maukuzon, N., Reynolds, J., Rosen, D.B.: Fuzzy ARTMAP: a neural network architecture for incremental supervised learning of analog multidimensional maps. IEEE Transactions on Neural Networks 3 (5). (1992) 698-713

14. Bezdek, J.C.: Pattern Recognition with Fuzzy Objective Function Algorithms. Plenum Press. New York (1981)

15. Comaniciu, D., Meer, P.: Mean shift: a robust approach toward feature space analysis. IEEE Transactions on Pattern Analysis and Machine Intelligence 24 (5). (2002) 603-619.

16. Nikolaou, N., Papamarkos, N.: Color segmentation of complex document images. International Conference on Computer Vision Theory and Applications. Setúbal, Portugal, (2006) 220-227

17. Zagoris, K., Papamarkos, N. and Koustoudis, I.: Color Reduction using the combination of the Kohonen Self-Organized Feature Map and the Gustafson-Kessel fuzzy algorithm. International Conference on Machine Learning and Data Mining MLDM 2007, (2007) 703-715.

18. Kohonen, T.: The self-organizing map. Proceedings of IEEE vol. 78, no.9. (1990) 1464-1480

19. Kohonen, T.: Self-Organizing Maps. 2nd Edition, Springer-Verlag. Berlin (1997)

20. Sagan, H.: Space-Filling Curves. Springer-Verlag. New York (1994)

21. Gustafson, E.E., Kessel, W.C. Fuzzy Clustering with a Fuzzy Covariance Matrix. Proc. 18th IEEE Conference on Decision and Control (IEEE CDC, San Diego, CA). Piscataway, NJ, USA (1979) 761-766 


\section{Vitae}

Konstantinos Zagoris received the Diploma in Electrical and Computer Engineering in 2003 from Democritus University of Thrace, Greece. He is currently a research and teaching assistant and studies towards the Ph.D. degree at the Department of Electrical and Computer Engineering, Democritus University of Thrace. His research interests include document retrieval, color image processing and analysis, document analysis and pattern recognition. He is a member of the Technical Chamber of Greece.

Nikos Papamarkos was born in Alexandroupoli, Greece, in 1956. He received his Diploma Degree in Electrical and Mechanical Engineering from the University of Thessaloniki, Thessaloniki, Greece, in 1979 and the Ph.D. Degree in Electrical Engineering in 1986, from the Democritus University of Thrace, Greece. From 1987 to 1990 Dr. Papamarkos was a Lecture, from 1990 to 1996 Assistant Professor, 1996-2003 Associate Professor in the Democritus University of Thrace where he is currently Professor since 2003. During 1987 and 1992 he has also served as a Visiting Research Associate at the Georgia Institute of Technology, USA. His current research interests are in digital signal processing, image processing, pattern recognition, neural networks and computer vision. Professor Nikos Papamarkos is a Senior Member of IEEE, Member of IAPR (Member of the Governing Board), Member of IEE and Member of the Greek Technical Chamber.

Ioannis Koustoudis received the Diploma in Electrical and Computer Engineering in 2001 and Ms Thesis in 2003 from Democritus University of Thrace, Greece. He is currently a research associate at the Department of Electrical and Computer Engineering, Democritus University of Thrace. His research interests include clustering and color image processing. He is a member of the Technical Chamber of Greece. 